\title{
One step procedure for screening and diagnosis of gestational diabetes mellitus by diabetes in pregnancy study group of India
}

\author{
Dwarakanath L., Hema K. R.*, Hemashree P.
}

Department of Obstetrics and Gynaecology, Shree Siddhartha Medical College, Tumkum, Karnataka, India

Received: 11 June 2019

Revised: 27 June 2019

Accepted: 16 July 2019

\section{*Correspondence:}

Dr. Hema K. R.,

E-mail: drhema78@gmail.com

Copyright: () the author(s), publisher and licensee Medip Academy. This is an open-access article distributed under the terms of the Creative Commons Attribution Non-Commercial License, which permits unrestricted non-commercial use, distribution, and reproduction in any medium, provided the original work is properly cited.

\begin{abstract}
Background: In the Indian context, screening for Diabetes is essential in all pregnant women, as the Indian women have an eleven-fold increased risk of developing glucose intolerance during pregnancy. For this, we need a simple procedure which is economical and feasible. Hence this study was undertaken as a screening as it is acceptable, economical and feasible to perform. Aim of this study was to assess the feasibility of one step procedure for screening and diagnosis of Gestational Diabetes Mellitus by Diabetes in Pregnancy Study Group of India (DIPSI).aim of the study was to study the occurrence of Gestational Diabetes mellitus, Tumkur, to assess the sensitivity and specificity of glucose challenge test, to assess the need for universal screening and to study the maternal and perinatal outcomes in patient with Gestational Diabetes Mellitus.

Method: Type of study was prospective study. this study included 200 pregnant women attending the antenatal OPD in Sri Siddhartha Medical College, Tumkur. Data collection was in a predesigned proforma. Pregnant women with 24-28 weeks of gestation were given 75 grams of oral glucose load, irrespective of their meal and venous blood sample drawn after 2 hours. If blood glucose value was $\geq 140 \mathrm{mg} / \mathrm{dl}$, the screening was considered as DIPSI positive. These patients underwent OGTT.

Results: Incidence of GDM was found to be $3.5 \%$ in the patients studied. $40 \%$ of cases did not have risk factors, hencethere is a need for universal screening. DIPSI was positive in 10 cases, of which 7 were OGTT positive. Patients were managed with diet and insulin. The maternal and perinatal outcome of pregnancy was good.

Conclusion: For universal screening, DIPSI performed irrespective of last meal timing with $75 \mathrm{~g}$ glucose load is a patient friendly approach. This method recommended by WHO serves both as a one-step screening and diagnostic procedure $\&$ is easy to perform besides being economical.
\end{abstract}

Keywords: Diabetes in pregnancy study group of india, Gestational Diabetes mellitus, Oral glucose tolerance test, World health organization

\section{INTRODUCTION}

Gestational diabetes mellitus is essentially the development of diabetes in pregnancy with a return to normal glucose tolerance after delivery.
Virtually all new cases of diabetes mellitus in pregnancy are a transient form of type II diabetes. A small proportion of cases of de novo diabetes are found to persist after pregnancy. Most of these are type II Diabetes Mellitus. However rarely Type I Diabetes Mellitus will arise during pregnancy simply as a matter of coincidence. $^{1}$ 
Pregnancy is considered to be a diabetogenic state characterized by exaggerated rate and amount of insulin release, associated with decreased sensitivity to insulin at cellular levels. Hormones like estrogen, progesterone, human placental lactogen, cortisol and growth hormone are anti insulinogenic. These hormones increase in mid pregnancy period and cause abnormal glucose tolerance in some women rendering them prone for gestational diabetes. ${ }^{2}$ "Carbohydrate intolerance of variable severity with the onset or first recognition during the present pregnancy". The definition applies irrespective of whether or not insulin is used for the treatment or the condition persists after pregnancy.

\section{Classification}

According to National Diabetes Data Group classification Type I - IDDM - Insulin Dependent diabetes

Type II - NIDM - Noninsulin dependent diabetes

Type III - GDM

This system is inadequate during pregnancy because it lacks further categorization according to the stability of the disease and to the presence or absence of target organ damage. The criteria are important in determining prognosis of the pregnancy.

ADA (American Diabetes Association) classified on the basis of etiology.

\section{Type I Diabetes}

\section{Type II Diabetes}

\section{Other specific type diabetes}

- $\quad$ Genetic defects of beta Cells

- Genetic defects in insulin action

- Disease of exocrine pancreas

- Endocrinopathies

- Drug or chemical induced

- Infections

- Immune mediated

\section{Gestational diabetes mellitus}

5. Impaired glucose tolerance (IGT) Impaired fasting glucose (IFG)

- $\quad$ IGT - 2 hrs GTT - >140 - <200 mg/dl

- $\quad$ IFG - FPG $>100-<126 \mathrm{mg} / \mathrm{dl}$

Women with a history of GDM are at increased risk of future diabetes, predominately type 2 diabetes, as are their children. The importance of GDM is that two generations are at risk of developing diabetes in the future. Besides any abnormal glucose tolerance during pregnancy also has adverse fetal outcome. ${ }^{3}$
Increasing maternal carbohydrate intolerance in pregnant without GDM is associated with a graded increase in adverse maternal and fetal outcomes. ${ }^{4}$ It is important to identify a pregnant woman with gestational diabetes mellitus because GDM is associated with significant metabolic alterations, increased perinatal mortality and morbidity, maternal morbidity and exaggerated long term morbidity among the mothers and their offspring. ${ }^{5}$

\section{Maternal fetal complications}

Risk of gestational diabetes for the Maternal and Fetus

\section{Fetal risks: perinatal}

- Macrosomia

- Intrauterine growth restriction

- Organomegaly (hepatomegaly, cardiomegaly)

- $\quad$ Birth trauma

- Shoulder dystocia

- Neonatal hypoglycemia

- Neonatal respiratory problems

- Hyperbilirubinemia

- Polycythemia

- $\quad$ Perinatal mortality

\section{Maternal risks: pregnancy / perinatal}

- Preeclampsia

- Polyhydramnios

- $\quad$ Operative delivery

Offspring risks: long term

- Obesity

- $\quad$ Type 2 diabetes

- Cardiovascular disease

- Impaired cognitive development

- Impaired motor function

Maternal risks: long term

- $\quad$ Type 2 diabetes

- Cardiovascular disease

\section{Perinatal complications}

Maternal hyperglycemia has an effect on fetal growth, glucose metabolism, iron metabolism, oxygenation and preparation for extra uterine life.

\section{METHODS}

\section{Source of data}

This is a prospective study conducted in SSMC Tumkur, for a period of one year from August 2014 to October 2016 , in all pregnant women attending OPD of OBG Department. 
The number of cases included in the study was 200 .

\section{Inclusion criteria}

Pregnant women attending antenatal OPD with gestational age between 24-28 weeks.

\section{Exclusion criteria}

Pregnant women diagnosed with diabetes prior to pregnancy i.e. Pre-gestational diabetes.

After informed consent, 200 pregnant women attending the OPD underwent detailed clinical examination as per proforma, irrespective of presence or absence of risk factors. 75 grams of glucose was dissolved in $300 \mathrm{ml}$ of water and the patient was asked to drink it over a five minute period, irrespective of time of the day and her last meal. After 2 hours of ingestion of glucose, venous blood was drawn. The plasma glucose was estimated by glucose oxidation and peroxidation (GOD-POD) method by EcoPak glucose kit. Glucose oxidase (GOD) converts glucose to gluconic acid. Hydrogen peroxide formed in this reaction, in the presence of peroxidase (POD), oxidatively couples with 4-aminoantipyrine and phenol to produce red quinoneimine dye. This dye has absorbance maximum at $505 \mathrm{~nm}(500-550 \mathrm{~nm})$. The intensity of the color complex is directly proportional to the concentration of glucose in the specimen. If plasma glucose value was $\geq 140 \mathrm{mg} / \mathrm{dl}$, the screening was considered as positive.

Patients with DIPSI value of $200 \mathrm{mg} / \mathrm{dl}$ or more were directly diagnosed as GDM without the need for OGTT. The DIPSI positive patients underwent diagnostic OGTT, by $100 \mathrm{gm}$ of glucose. Three days prior to OGTT test, patients were asked to take normal unrestricted diet. After overnight fasting of 8-14 hours, a fasting blood sample was drawn, following which $100 \mathrm{gm}$ of glucose dissolved in $300-400 \mathrm{ml}$ of water was given orally.

Thereafter venous glucose plasma levels were assessed hourly for three hours.

Patients were diagnosed as GDM by Carpentar and Coustan values.

Those diagnosed as GDM were admitted, evaluated, treated and followed till delivery and also after puerperium. Maternal and perinatal outcomes were studied.

Data was entered in Microsoft excel, graphs were drawn using Microsoft and Microsoft excel. Data was analysed using SPSS software version.14.

Statistical tests like Fischer exact test was used.

\section{RESULTS}

Among 200 patients, all the patients accepted the DIPSI test readily and no adverse effects were observed. DIPSI was positive in 10 cases and of which 7 were OGTT positive. It makes DIPSI $100 \%$ sensitive and $98.44 \%$ specific with positive predictive value $70 \%$. In this study there is gradual increase in prevalence of DIPSI positive cases with increase in age. There was higher risk of developing DIPSI positive with higher BMI. in this study, the risk factors for positive DIPSI of $>35 \%$ were age $>30 y$ rs, family history of diabetes mellitus, previous pregnancy loss PCOS, twins, BMI $>25$.

It was found that DIPSI was positive in $40 \%$ of cases without risk factors. Therefor universal screening is needed. The mode of delivery in DIPSI positive cases more than $50 \%$ of DIPSI positive cases was LSCS. Complication of pregnancy in DIPSI positive cases were as follows,PIH in two cases like infection. PIH was present in 2 cases, poly hydroaminos in one case and infection in one case. DIPSI was positive equally in primi and multigravida five in each, case this study had 2 patients as DIPSI positive constituting $15.38 \%$ out of 13 patients between 30-34 years. (our study had 2patients DIPSI positive constituting $15.38 \%$ among out of 13 people between $30-34 y$ rs in this study population 2 of them where DIPSI positive

\section{Table 1: Distribution of GDM cases according to} risk factors.

\begin{tabular}{|lllll|}
\hline Risk factors & Total & GDM & $\%$ & P value \\
\hline Age $>30 y r s$ & 19 & 2 & 10.52 & $>0.05$ \\
\hline Family H/o & 10 & 4 & 40 & $<0.05$ \\
\hline Previous LSCS & 9 & 1 & 11.11 & $>0.05$ \\
\hline PCOD & 1 & - & - & - \\
\hline Twin & 2 & - & - & - \\
\hline BMI $>25$ & 8 & 3 & 37.5 & $<0.05$ \\
\hline
\end{tabular}

$\mathrm{P}$ value $<0.05$ was statistically significant in Family history and BMI $>25$.

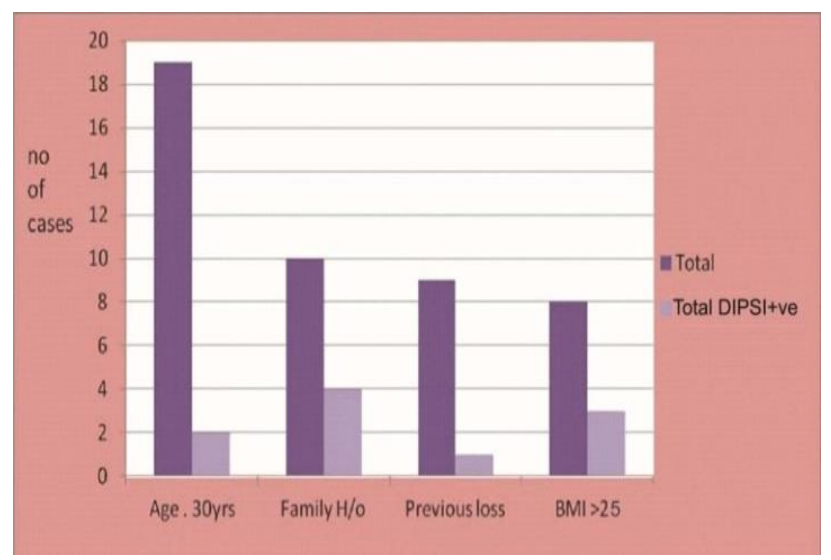

Figure 1: Association of risk factors with GDM. 
Mode of delivery was LSCS (28.52), 2 of them had elective LSCS and 2 of them had emergency LSCS among 7 who were DIPSI positive. There was no macrosomia in those who were DIPSI positive, 3 babies weighed between 3 to $3.5 \mathrm{~kg}$ and 2 were between 3.6 to 4 $\mathrm{kg}$ remaining 2 were less than $3 \mathrm{kgs}$. Neonatal outcome, all babies did well except only 2 babies of which mother was DIPSI positive, one had hypoglycemia and one baby had neonatal death.

Table 2: Mode of delivery in GDM cases.

\begin{tabular}{|lll|}
\hline Mode of delivery & No. of cases & Percentage \\
\hline Vaginal spontaneous & 2 & 28.52 \\
\hline Vaginal Induced & 1 & 14.26 \\
\hline Elective LSCS & 2 & 28.52 \\
\hline Emergency LSCS & 2 & 28.52 \\
\hline
\end{tabular}

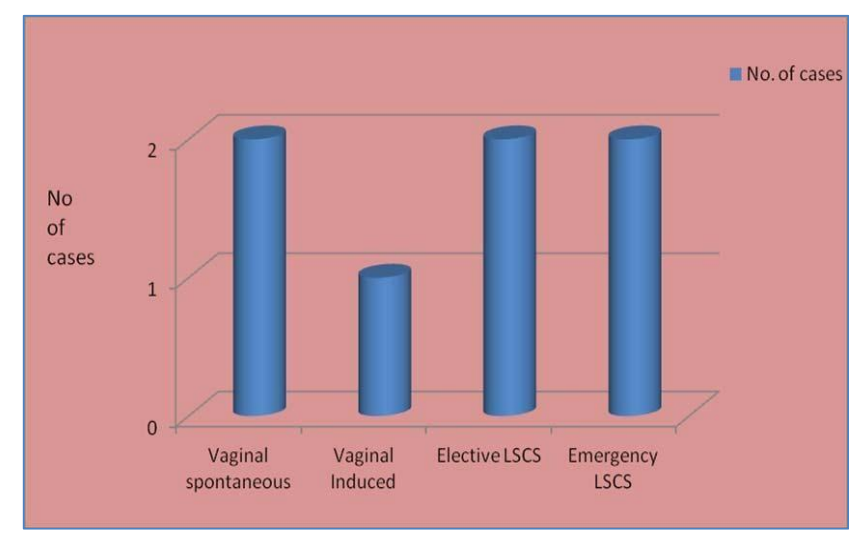

Figure 2: Mode of delivery in GDM cases.

\section{DISCUSSION}

"Gestational diabetes mellitus is a Carbohydrate intolerance of variable severity with the onset or first recognition during the present pregnancy". GDM is associated with important prenatal and long term health risks and many of the risks increases in relation to the severity of maternal hyperglycemia.

Chinese $7.3 \%$ and Hispanic $4.2 \%$ women than for blacks. Ferrara et al, also showed the incidence at $7.5 \%$ in Asians, 5.6\% in Hispanics, 4\%, African American. Engelgau et al, reported that whilst GDM complicates about $5 \%$ of all pregnancies, but the rate of occurrence can range up to $14 \%$ depending on the population subgroup. ${ }^{6}$

The incidence of diabetes in the world at large $\&$ in India in particular is on the raise. Indian population, is ethnically prone to high prevalence of type II DM. In such population, incidence of GDM is also high. Incidence of GDM ranges from $0.2 \%-12 \%$ depending on the population studied. In Indian context our women have 11 fold increase in rate of developing GDM. ADA noticed that $7 \%$ of all pregnancies are complicated by GDM \& in this study the incidence is $3.5 \%$.

The sensitivity of the test was $100 \%$ in Anjalakshi et al, and also in this study, so were the sensitivity test with 75 grams glucose. The positive predictive value was high compared to Carpentar and Coustan`s study indicating that screening with 75 grams glucose is a more sensitive test in detecting GDM. Sheshiah et al report from their study that using $75 \mathrm{~g}$ glucose at $2 \mathrm{~h}$ plasma glucose $\geq 140$ $\mathrm{mg} / \mathrm{dl}$ as one step procedure is simple and economical, particularly for the countries ethnically prone to high prevalence of GDM. ${ }^{7}$ Anjalakshi et al, in their study of 800 pregnant women diagnosed as GDM by 75 g GCT glucose, irrespective of the last meal timings, found no statistically significant difference $(\mathrm{P}>0.005)$ between the plasma glucose levels of GCT and WHO GTT performed in the GDM and the normal glucose tolerance pregnant women. ${ }^{8}$

Esakoff et al, also examined if the screening criteria should be modified depending on the ethnicity of the subject being tested. Bonomo et al, recommended the threshold of $140 \mathrm{mg} / \mathrm{dl}$ as a cut off point for screening GDM, as the lower threshold values are associated with high false positive rate, increasing the economic burden. Taking $140 \mathrm{mg} / \mathrm{dl}$ as a cutoff point, only $14 \%$ require OGTT whereas, $23 \%$ require OGTT with $130 \mathrm{mg} / \mathrm{dl}$ as a cutoff point. If the prevalence of GDM is high in a particular population group, a cutoff point of $130 \mathrm{mg} / \mathrm{dl}$ is a reasonable threshold level. And a higher false positive rate is also acceptable. ${ }^{9,10}$

Established risk factors for GDM are advanced maternal age, obesity and family history of diabetes. Sheshiah et al, noted increase in the prevalence of GDM in their study and attributed it to increased BMI, as high maternal weight is associated with a substantially higher risk of GDM. ${ }^{11}$

Jang et al found that the GDM women were older, had higher pre pregnancy weight, higher BMI, higher parities and higher frequencies of known diabetes in the family. Of all the independent risk factors for GDM, BMI emerged as a modifiable risk factor. GDM women have high risk of developing diabetes in the future. They are the ideal group to be targeted for lifestyle modification or pharmacologic intervention in order to delay or postpone the onset of overt diabetes. Hence an important public health priority in the prevention of diabetes is to address maternal health both during antenatal and post-partum period. ${ }^{12}$

Solomon CG et al study showed advance maternal age, family history of diabetes mellitus, nonwhite ethnicity, higher BMI, weight gain in early adulthood and cigarette smoking predicts increased GDM risk and this observations facilitate the identification of women at particular risk for GDM and suggests potential strategies for reducing the risk even before a women becomes 
pregnant such as avoiding substantial weight gain and smoking. ${ }^{13}$

Maternal age is an established risk factor for gestational diabetes mellitus (GDM), but there is no consensus on the age above which there is significantly increased risk of GDM. In the literature, the lowest cut off is 25 years, as recommended by the American Diabetes Association. Though the incidence in different age groups varies, in all studies, there is an increase in incidence of GDM as the age advances.

Terence $\mathrm{T}$ Lao et al, finding indicates that the risk of GDM becomes significantly and progressively increased from 25 years onwards. This supports the American Diabetes Association recommendation on the use of age 25 years as the cutoff for screening and the observation that maternal age 25 years is the factor most predictive of GDM. In clinical practice, maternal age of 25 years should be adopted instead of 35 years or 40 years as a risk factor for the development of GDM. ${ }^{14}$

The most significant maternal risk with GDM is the 35 $50 \%$ probability of developing type II diabetes later in life. Older studies indicated a significant increase in incidence of PIH but recent studies such as Naylor et al, questions these findings. ${ }^{15}$ Polyhydramnios occurs frequently in GDM particularly when the fetus is macrosomic.

In this Study, polyhydramnios was associated with increased birth weight. So tight control of blood sugar level was needed. The incidence of cesarean section is higher than in the non-diabetic population, but possibly this is a consequence of the diagnosis and not the condition. Naylor et al found that there was an increased rate of cesarean delivery in treated diabetic women whether the infant was macrosomic or not. Macrosomia is an important complication of GDM, it increased the risk of cesarean delivery there was no difference in the rates of cesarean delivery between large and normal weight infants among treated diabetic women. Macrosomia was not documented in this study due to immediate management with diet (57.7\%) and diet+insulin (42.86\%). In this study, two cases had a birth weight of $>3.5 \mathrm{~kg}$. Probably because of appropriate management of diabetes. Naylor et al suggests that while medical factors and infant size may account for some of the increased risk of cesarean delivery among diabetic women, practice patterns and physician referrals to high risk care may be large factor. ${ }^{15}$

In this study incidence of LSCS is $>50 \%$, is higher as compared to other studies. Emergency LSCS is higher than Elective LSCS \& main indications are fetal distress and failure of progress of labor. Schafer et al, reported an increased incidence of anomalies commonly associated with type I diabetes mellitus in women with either GDM or type II diabetes mellitus. The anomalies were associated with fasting hyperglycemia and elevated
HbA1C values and may represent women with undiagnosed pregestational type II diabetes mellitus. Anamolies are not found in this study because preexisting diabetes have been excluded. Anamolies are usually seen in preexisting diabetes rather than GDM. ${ }^{16}$

$\mathrm{O}^{\text {ee }}$ Sullivan et al, reported that the perinatal mortality was $6.4 \%$ among GDM when compared to normal controls. Abell et al, also noted that with treatment, perinatal mortality in Mercy maternity hospital fell down to $3.9 \%$ from $14.3 \%$ In this study there was 1 neonatal deaths. These patients had good glycemic control. ${ }^{17}$ Postnatal follow up after 6 weeks with OGTT was done and were found to be OGTT negative. ${ }^{17}$

\section{CONCLUSION}

Glucose Challenge test with $75 \mathrm{~g}$ glucose at 2 hours is highly sensitive in detecting GDM. DIPSI performed irrespective of the last meal timings is a patient friendly approach and causes least disturbance in the pregnant women's routine activities. For universal screening one step versus two step, the two step procedure of screening with GCT and then diagnosing GDM based on the cut off values with $100 \mathrm{~g}$ or $75 \mathrm{~g}$ OGTT is not practical as the pregnant women have to visit the clinic atleast twice and the number of blood samples drawn vary from 3 to 5 . It is suggested $75 \mathrm{~g}$ OGTT load and diagnosing GDM if 2hour PPG is $\geq 140 \mathrm{mg} / \mathrm{dl}$ as recommended by WHO. Hence, DIPSI method serves both as one step and a diagnostic procedure and is easy to perform besides being economical. Gestational Diabetes Mellitus can be present in patients without risk factors, hence the need for universal Screening. Timely intervention with diet, insulin therapy, patient education and team approach for the

Funding: No funding sources Conflict of interest: None declared

Ethical approval: The study was approved by the Institutional Ethics Committee

\section{REFERENCES}

1. Judith Hyde, Emma Treloar, Robert Fox. Progress in OB\&G. Publisher Edinburgh: Elsevier, $16^{\text {th }}$ Vol. 2005:52-72.

2. Danilenko-Dixon DR, Van Winter JT, Nelson RL, Ogburn Jr PL. Universal versus selective gestational diabetes screening: application of 1997 American Diabetes Association recommendations. Am J Obstet Gynecol. 1999 Oct 1;181(4):798-802.

3. Dornhorst A, Rossi M. Risk and prevention of type 2 diabetes in women with Gestational diabetes. Diabetes Care. 1998;21(2):B43-9.

4. Sermer M, Naylor CD, Farine D, Kenshole AB. The Toronto Tri Hospital Gestational diabetes Project- A preliminary review. Diabetes Care. 1998;21(2):B3342. 
5. First International Workshop- Conference on Gestational Diabetes Mellitus Summary and recommendations. Diabetes Care. 1980;3:499-501.

6. Ferrara A, Hedderson MM, Quesenberry CP, Selby JV. Prevalence of gestational diabetes mellitus detected by the national diabetes data group or the carpenter and coustan plasma glucose thresholds. Diabetes Care. 2002Sep1;25(9):1625-30.

7. Sheshiah V, Balaji V, Balaji MS et al. One step for screening and diagnosis of gestational diabetes mellitus. J Obstet Gynecol India. 2005;55:525-9.

8. Anjalakshi, Balaji, Madhuri et al.A Single procedure to diagnose gestational diabetes mellitus. Acta Diabetol. 2009 Mar1;46(1):51-4.

9. Esakoff T, Cheng Y, Caughey A. Screening for GDM: different cutoffs for different ethnicities? Am J Obstet Gynecol. 2005Sep1;193(3):1040-4.

10. Bonomo M, Gandini ML, Mastropasqua A, Begher C, Valentini U, Faden D, et al. for the Definition of Screening Methods for Gestational Diabetes Study Group of the Lombardy Section of the Italian Society of Diabetol. Am J Obstet Gynecol. 1998;179(1):17985 .

11. Seshiah V, Balaji V, Balaji MS, Paneerselvam A, Arthi T, Thamizharasi M, et al. Gestational diabetes mellitus manifests in all trimesters of pregnancy. Diabetes research and clinical practice. 2007 Sep 1;77(3):482-4.

12. Jang HC, Cho NH, Jung KB, Oh KS, Dooley SL, Metzger BE. Screening for gestational diabetes mellitus in Korea. Int J Gynecol Obstet. 1995 Nov 1;51(2):115-22.

13. Solomon CG, Willett WC, Carey VJ, Rich-Edwards J, Hunter DJ, Colditz GA, et al. A prospective study of pregravid determinants of gestational diabetes mellitus. JAMA. 1997 Oct 1;278(13):1078-83.

14. Terence T. Lao, MD, Lai-Fong Ho, MSC, Ben C.P. Chan, and Wing- Cheong Leung. Maternal Age and Prevalence of Gestational Diabetes Mellitus. Diabetes Care. 2006;29:948-9,

15. Naylor CD, Sermer M, Chen et al. Cesarean delivery in relation to birth weight gestational glucose tolerance: pathophysiology or practice style? JAMA. 1996 Apr 17;275(15):1165-70.

16. Coustan DR, Carpenter MW, O'Sullivan PS, Carr SR. Gestational diabetes: predictors of subsequent disordered glucose metabolism. Am J Obstet Gynecol. 1993 Apr 1;168(4):1139-44.

17. Abell DA, Beischer NA, Wood C. Routine testing for gestational diabetes, pregnancy, hypoglycemia \& fetal growth retardation \& results of treatment. J Perinatal Medic. 1976Jan1;4:197-212.

Cite this article as: Dwarakanath L, Hema KR, Hemashree P. One step procedure for screening and diagnosis of gestational diabetes mellitus by diabetes in pregnancy study group of India. Int J Reprod Contracept Obstet Gynecol 2019;8:3344-9. 